\title{
Macrophages ameliorate bone marrow inflammatory injury and promote hematopoiesis in mice following hematopoietic stem cell transplantation
}

\author{
JIANLIN QIAO ${ }^{1-3^{*}}$, LU LIU $^{1 *}$, YUAN XIA ${ }^{1 *}$, WEN JU ${ }^{1-3}$, PINGPING ZHAO $^{1}$, YAN JIANG ${ }^{1}$, MINGFENG LI $^{1}$, WEN LI $^{1}$, \\ LAN DING $^{1}$, YULU WU ${ }^{1}, \mathrm{KUNMING}^{2}$, DEPENG $\mathrm{LI}^{2}, \mathrm{XI} \mathrm{ZHANG}^{4}, \mathrm{KAILIN} \mathrm{XU}^{1-3^{*}}$ and LINGYU ZENG ${ }^{1-3^{*}}$ \\ ${ }^{1}$ Blood Diseases Institute, Xuzhou Medical University; ${ }^{2}$ Department of Hematology, The Affiliated Hospital of \\ Xuzhou Medical University; ${ }^{3}$ Key Laboratory of Bone Marrow Stem Cell, Xuzhou, Jiangsu 221002; \\ ${ }^{4}$ Department of Hematology, Xinqiao Hospital, Third Military Medical University, Chongqing 400037, P.R. China
}

Received November 6, 2017; Accepted March 13, 2018

DOI: $10.3892 / \mathrm{etm} .2018 .6209$

\begin{abstract}
Bone marrow macrophages have been demonstrated to serve a critical role in promoting maintenance and retention of hematopoietic stem cells (HSCs). Our previous study indicated increased macrophages infiltration in bone marrow after HSC transplantation (HSCT). However, it is not well understood whether macrophages affect hematopoietic reconstitution after HSCT. The present study aimed to investigate the role of macrophages in hematopoietic reconstitution after HSCT. $\mathrm{BALB} / \mathrm{c}$ mice were divided into HSCT, HSCT+Clodronate Liposomes, HSCT+PBS Liposomes, HSCT+RS102895 and HSCT+Vehicle groups and sacrificed on day 7, 14, 21, 28 and 35 after HSCT. Analysis was performed to detect the changes of bone marrow pathology by H\&E staining and the number of macrophages was assessed by immunohistochemical staining and western blot analysis. The number of c-kit ${ }^{+} \mathrm{sca}-\mathrm{I}^{+}$and c-kit ${ }^{+}$ was measured by flow cytometry. Mice with a depletion of bone marrow macrophages displayed significantly reduced overall survival, delayed hematopoietic recovery, a reduced number of hematopoietic stem/progenitor cells and bone marrow cells as well as exaggerated bone marrow injury. However, compared with the HSCT+Vehicle group, mice with an increased number of bone marrow macrophages exhibited no difference of
\end{abstract}

Correspondence to: Dr Lingyu Zeng, Blood Diseases Institute, Xuzhou Medical University, 84 West Huaihai Road, Quanshan, Xuzhou, Jiangsu 221002, P.R. China

E-mail: zengly2000@163.com

Professor Kailin Xu, Department of Hematology, The Affiliated Hospital of Xuzhou Medical University, 99 West Huaihai Road, Quanshan, Xuzhou, Jiangsu 221002, P.R. China

E-mail: 1ihmd@163.com

*Contributed equally

Key words: macrophages, hematopoietic stem cell transplantation, bone marrow injury, hematopoietic reconstitution overall survival, had accelerated hematopoietic reconstitution, a higher number of hematopoietic stem/progenitor cells and bone marrow cells and ameliorated bone marrow injury. In conclusion, the present study indicated that bone marrow macrophages serve a protective role in bone marrow injury and may promote hematopoiesis in mice after HSCT, suggesting manipulation of macrophages may be a novel strategy for improving the efficacy of HSCT.

\section{Introduction}

Hematopoietic stem cell transplantation (HSCT) is a potentially effective strategy for treating several hematological malignancies, such as leukemia, multiple lymphoma, other disorders of blood and immune system $(1,2)$. Successful engraftment of hematopoietic stem cells (HSCs) in bone marrow followed by expansion into mature hematopoietic lineages is the prerequisite for effective HSCT $(3,4)$. Delayed or failed engraftment of HSCs (also called engraftment syndrome) will significantly impede hematopoietic reconstitution and reduce the treatment efficiency, resulting in higher incidence of mortality and morbidity in patients after HSCT (5).

HSCs Engraftment is largely dependent on the bone marrow environment, as HSCs reside in a highly specialized microenvironment or niche in the bone marrow, which is consisted of supporting cells, such as endothelial cells, lipocytes, fibroblasts, reticulocytes, and osteoblasts $(6,7)$. By interacting with corresponding receptors expressed on HSCs, soluble factors, molecules and ligands, which are produced from these supporting cells, regulate the self-renewal, proliferation, differentiation of HSCs (8). In the settings of HSCT, bone marrow is severely damaged due to pre-conditioning regimen treatment, leading to apoptosis of supporting cells, which would activate the mononuclear phagocyte system (MPS) comprising bone marrow progenitors, blood monocyte and tissue macrophages, resulting in rapid clearance of these apoptotic or dead cells (9).

Bone marrow microenvironment or niche is highly complex and previous studies add to this complexity through demonstrating a pivotal role of bone marrow mononuclear 
phagocytes in promoting maintenance and retention of HSCs (10-12). Using monocyte and macrophage conditional depletion models, Chow et al demonstrated that decreased bone marrow mononuclear phagocytes led to reduced bone marrow CXCL12 levels (CXCL12-CXCR4 is a critical niche retention signal), the selective downregulation of HSC retention genes in $\mathrm{Nestin}^{+}$niche cells, and subsequent egress of HSCs/progenitors to the bloodstream (10). In addition, bone marrow macrophages are also demonstrated to be required for the maintenance of endosteal HSC niches in vivo and loss of macrophages initiates a cellular cascade that ultimately leads to the mobilization of functional HSCs with repopulating activity which was associated with a decrease in transcripts of CXLC12, angiopoietin 1 (Ang-1), stem cell factor (SCF) in both bone marrow and endosteal stroma (12). Furthermore, bone marrow monocytes and macrophages with high expression of $\alpha$-smooth muscle actin were capable to maintain hematopoietic stem/progenitor cells and protect them from exhaustion during alarm situations (13).

Our previous study demonstrated increased infiltration of inflammatory cells (neutrophils and macrophages) into bone marrow in mice following HSCT, accompanied with increased secretion of several pro-inflammatory cytokines, including TNF- $\alpha$, IL-1 $\beta$ and IL-18 (14). Infiltrated macrophages can not only exaggerate bone marrow inflammatory injury through secretion inflammatory cytokines (15), but also can exert their function on the clearance of these apoptotic or dead cells (16), providing space for transplanted HSCs. Considering the importance of macrophage in bone marrow microenvironment, whether macrophages affect hematopoietic reconstitution after HSCT remains to be elucidated. In the present study, we aimed to evaluate the role of macrophage in hematopoietic reconstitution in mice after HSCT and found macrophage plays a protective role in bone marrow inflammatory injury and promotes hematopoietic reconstitution.

\section{Materials and methods}

Materials. Rabbit monoclonal to CD11b antibody was from Abcam (Cambridge, MA, USA). PE-conjugated anti-mouse CD80 antibody was purchased from eBioscience (San Diego, CA, USA). Alexa Fluro488-conjugated anti-mouse CD206 antibody was purchased from BioLegend (San Diego, CA, USA). PE-CY7-conjugated anti-mouse c-kit and APC-conjugated anti-mouse Sca-1 antibody were from BD Biosciences (San Jose, CA, USA). Clodronate Liposomes (Clo-Lipo) and PBS Liposomes (PBS-Lipo) were purchased from Liposoma B.V. (Amsterdam, The Netherlands). RS102895 was purchased from Tocris Bioscience (Ellisville, MO, USA).

Animals and treatment. All experimental procedures involving animals were approved by the Ethic Committee of Xuzhou Medical University (Xuzhou, China).

C57BL/6 and BALB/c mice, aged 8-10 weeks and weighed 24-28 g, were purchased from SLAC Laboratory Animal Co., Ltd. (Shanghai, China). The mice were housed in SPF grade environment in the Experimental Animal Center of Xuzhou Medical University.

$\mathrm{BALB} / \mathrm{c}$ mice were divided into five groups: HSCT $(n=16)$, HSCT+Clo-Lipo $(n=20)$, HSCT+PBS-Lipo $(n=20)$,
HSCT+RS102895 (n=20) and HSCT+Vehicle (n=20). HSCT mice model was established as previously described (14). Briefly, BALB/c mice received lethal irradiation with 7.5 Gy followed by infusion of $2 \times 10^{7}$ bone marrow mononuclear cells isolated from the C57BL/6 mice. For injection of Clo-Lipo or PBS-Lipo, $200 \mu 1$ Clo-Lipo or PBS-Lipo was administrated via tail vein on day 1 after HSCT with $100 \mu$ l injection per 3 days in the following periods. Regarding injection of RS102895, $0.15 \mathrm{mg}$ RS102895 was intraperitoneally injected into mice after HSCT every other day. Meanwhile, mice from Vehicle group received equal volume and concentration of DMSO after HSCT. Normal health mice without any treatment were served as a control.

$H \& E$ and immunohistochemical staining. On D7, 14, 21, 28 and 35 after HSCT, mice were sacrificed and bilateral femur and tibia were isolated, followed by fixation in formaldehyde solution, dehydrated, waxed, and sliced into $4 \mu \mathrm{m}$ thickness. After that, H\&E staining was performed and the pathologic changes of bone marrow were evaluated by a light microscope.

$3 \% \mathrm{H}_{2} \mathrm{O}_{2}$ was added into the bone marrow slices and incubated at room temperature followed by blockage by $5 \%$ goat serum. After that, the slices were incubated with anti-mouse CD11b antibody and subsequent with HRP-conjugated anti-rabbit secondary antibody. At the end point, color was developed using 3, 3'-diaminobenzidine. The number of CD11b positive cells in bone marrow was counted in 10 consecutive fields under high-power fields (x40) and represented as cell number per $\mathrm{mm}^{2}$.

Western blot analysis. Proteins were extracted from bone marrow cells of mice, separated on $10 \%$ SDS-PAGE, and transferred to an NC membrane. The membranes were incubated with rabbit anti-mouse CD11b antibody and then incubated with HRP-conjugated anti-rabbit secondary antibody. Membranes were visualized using enhanced chemiluminescence. $\beta$-actin was used as a control. Protein expression was quantified using Image $\mathbf{J}$ software. The results were represented as a ratio to $\beta$-actin.

Measurement of HSCs and progenitor cells. At indicated time point, bone marrow cells were isolated to measure the number of cells with c-kit ${ }^{+} \mathrm{sca}-1^{+}$, c-kit ${ }^{+}$by flow cytometry using anti-mouse c-kit ${ }^{+}$and sca- $1^{+}$antibodies $(17,18)$.

Statistical analysis. At least 3 independent experiments were performed for each assay. Data were analyzed by GraphPad Prism software (version 6.0; GraphPad Software, Inc., La Jolla, CA, USA) and represented as mean \pm SD. One-way ANOVA followed by Newman-Keuls multiple comparison post hoc analysis was used for comparison of one parameter at different time points after HSCT. Two-way ANOVA followed by Bonferroni's post hoc test was performed for comparison of differences between multiple groups over time. $\mathrm{P}<0.05$ was considered to indicate a statistically significant difference.

\section{Results and Discussion}

As shown in Fig. 1, bone marrow inflammatory damage was observed after transplantation with the most severe injury on 



Days after HSCT

Figure 1. H\&E staining of bone marrow and macrophages infiltration after HSCT. (A) At indicated time points after HSCT, mice were sacrificed and bilateral femur and tibia were isolated for analysis of bone marrow pathology by H\&E staining (magnification, x400). Bone marrow cells were also extracted for analysis of the expression of CD11b (a macrophage marker) by western blot (B) and macrophage polarization $(\mathrm{M} 1 / \mathrm{M} 2)$ by flow cytometry $(\mathrm{C})$. ${ }^{*} \mathrm{P}<0.05$ ( $\left.\mathrm{n}=4\right)$. ${ }^{*} \mathrm{P}<0.05$ and ${ }^{* *} \mathrm{P}<0.01$ compared with control or as indicated. NS, not significant; HSCT, hematopoietic stem cell transplantation.

D7 showing hemorrhage and cavity. On D14, hemorrhage still existed with lots of inflammatory cells infiltration into bone marrow. Afterwards, hemorrhage and cavity were reduced from D21 with increased bone marrow cells and ameliorated damage. The above pathological changes were almost recovered to normal on D35. Western blot analysis showed numbers of macrophages in bone marrow were increased after HSCT reaching a peak on D14 followed by a gradual decrease in the following periods, consistent with our previously study (14). In addition, infiltrated macrophage polarization was also measured and showed significantly higher number of M1 macrophage $\left(\% \mathrm{CD} 80^{+}\right)$and lower number of M2 macrophage $\left(\% \mathrm{CD}^{206^{+}}\right)$on D14 post transplantation. However, the number of M1 macrophage decreased and M2 macrophage increased on D35.

Considering increased number of macrophages in bone marrow during bone marrow inflammation aftertransplantation, Clo-Lipo was used to deplete macrophages (19) and RS102895 (inhibition chemotaxis of macrophages to peripheral) was used to increase macrophages in bone marrow to investigate the role of macrophages in HSCT. As seen in Fig. 2A, number of macrophages in bone marrow was dramatically reduced after
Clo-Lipo treatment as demonstrated by immunohistochemistry staining of bone marrow compared with PBS-Lipo $(\mathrm{P}<0.01)$. Consistently, western blot analysis also showed reduced CD11b (a pan-macrophage marker) expression in bone marrow (Fig. 2B). Meanwhile, after RS102895 treatment, number of macrophages in bone marrow was increased as demonstrated by immunohistochemistry staining (Fig. 2C) and western blot (Fig. 2D) compared with Vehicle $(\mathrm{P}<0.01)$.

Peripheral blood was drawn from Clo-Lipo-treated mice or control mice for analysis the effect of macrophages on peripheral hematopoietic reconstitution. As seen in Fig. 3A, numbers of white blood cell, red blood cell and platelet were significantly lower in mice treated with Clo-Lipo compared with PBS-Lipo on D14 post transplantation. However, no differences were observed on D35 between these two groups. Different to Clo-Lipo-treated mice, mice after RS102895 treatment displayed accelerated hematopoietic reconstitution which was demonstrated by higher number of white blood cells and platelets on D14 post HSCT (Fig. 3B). Taken together, these data demonstrated that macrophages might play an important role in promotion of peripheral hematopoietic reconstitution after HSCT. However, the exact mechanism 

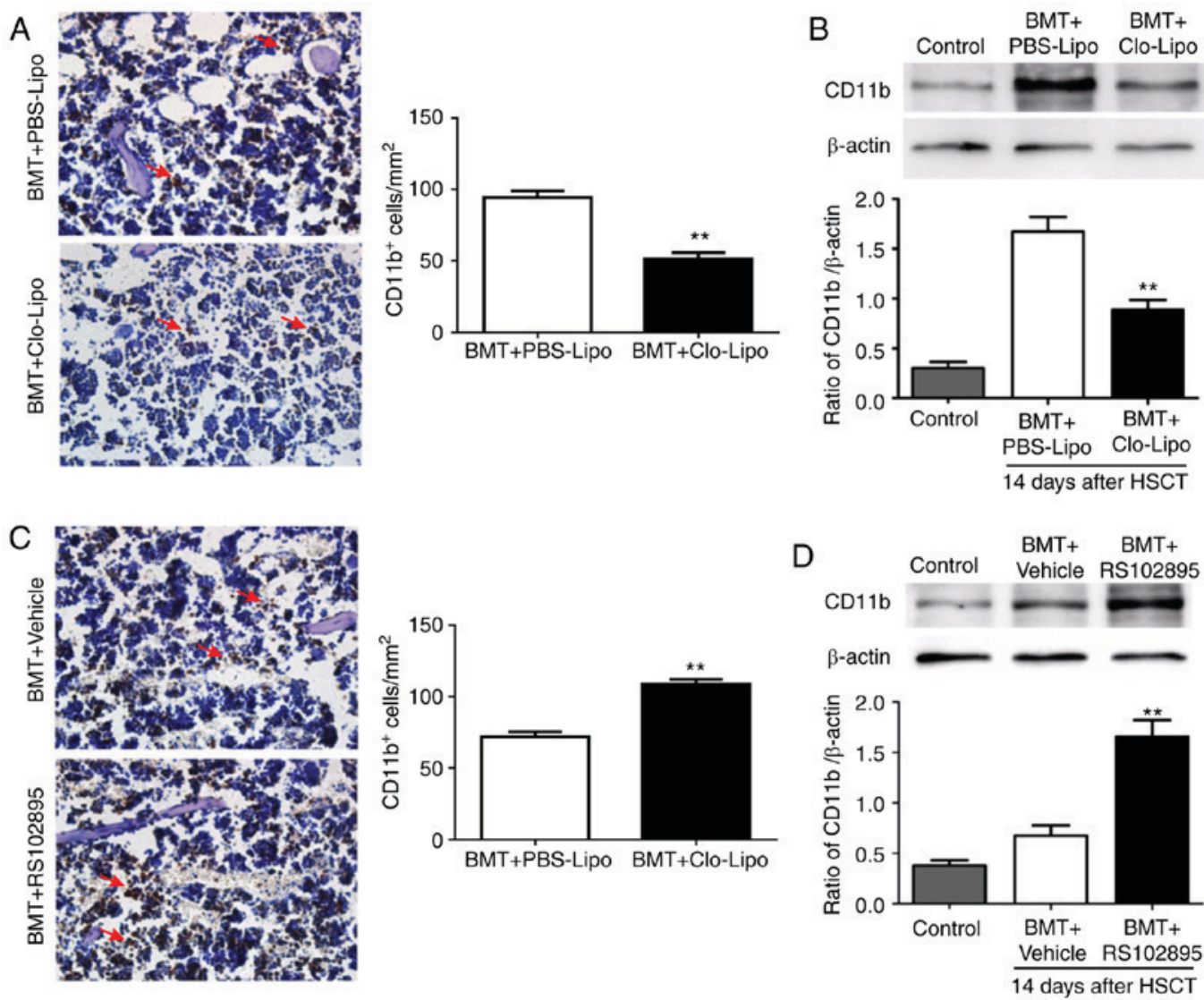

Figure 2. Bone marrow macrophages after Clo-Lipo (A and B) or RS102895 (C and D) treatment following HSCT. Bone marrow slices were incubated with $3 \% \mathrm{H}_{2} \mathrm{O}_{2}$ at room temperature and blocked with 5\% goat serum. Then the slices were incubated with anti-mouse CD11b antibody for analysis of macrophages by immunohistochemical staining (A and C) (magnification, x200). Bone marrow cells were also isolated for analysis of CD11b expression by western blot analysis (B and D). All the data were acquired from at least 3 independent experiments. Compared with BMT+PBS-Lipo or BMT+Vehicle, ${ }^{* *} \mathrm{P}<0.01$ ( $\mathrm{n}=3$ ). Arrows indicated the macrophages (positive staining of CD11b). Clo-Lipo, Clodronate Liposomes; HSCT, hematopoietic stem cell transplantation; PBS-Lipo, PBS Liposomes.

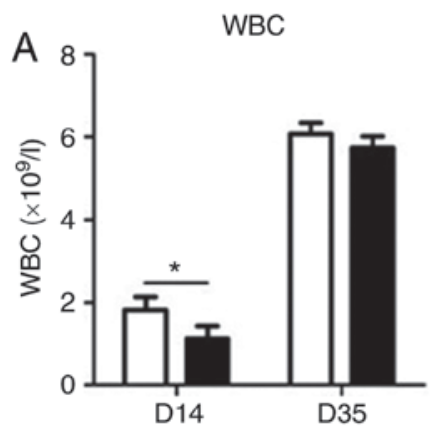

Days after HSCT

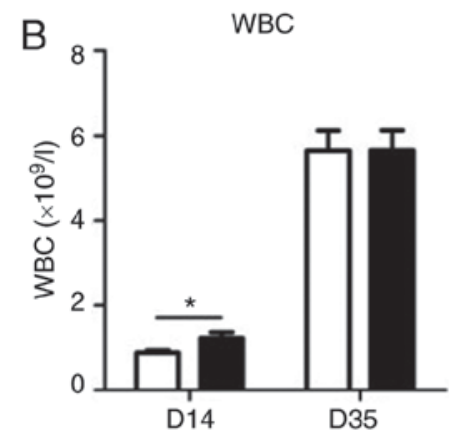

Days after HSCT

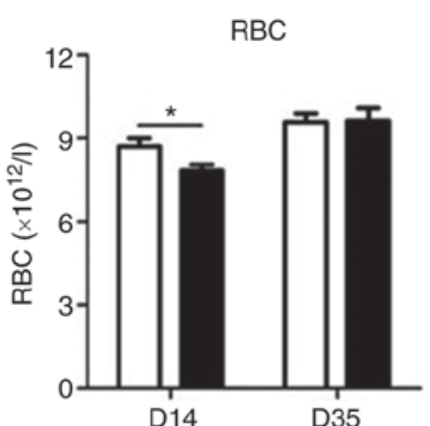

Days after HSCT

$\mathrm{RBC}$

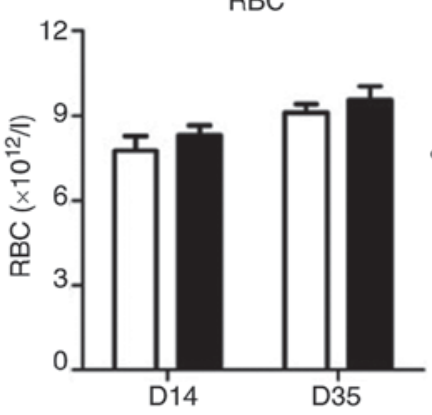

Days after HSCT

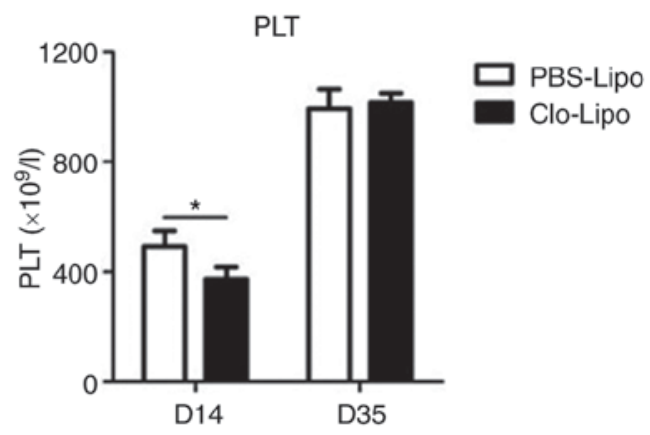

Days after HSCT

PLT

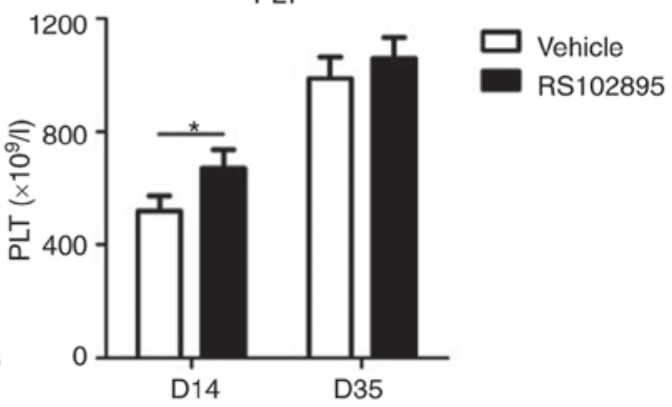

Days after HSCT

Figure 3. Hematopoietic reconstitution in mice after treatment with Clo-Lipo or RS102895 after HSCT. At indicated time points after HSCT, peripheral blood of mice treated with Clo-Lipo (A) or RS102895 (B) was drawn for analysis of the number of WBC, RBC and PLT. "P<0.05 ( $\mathrm{n}=4$ ). Clo-Lipo, Clodronate Liposomes; HSCT, hematopoietic stem cell transplantation; WBC, white blood cells; RBC, red blood cells; PLT, platelets. 

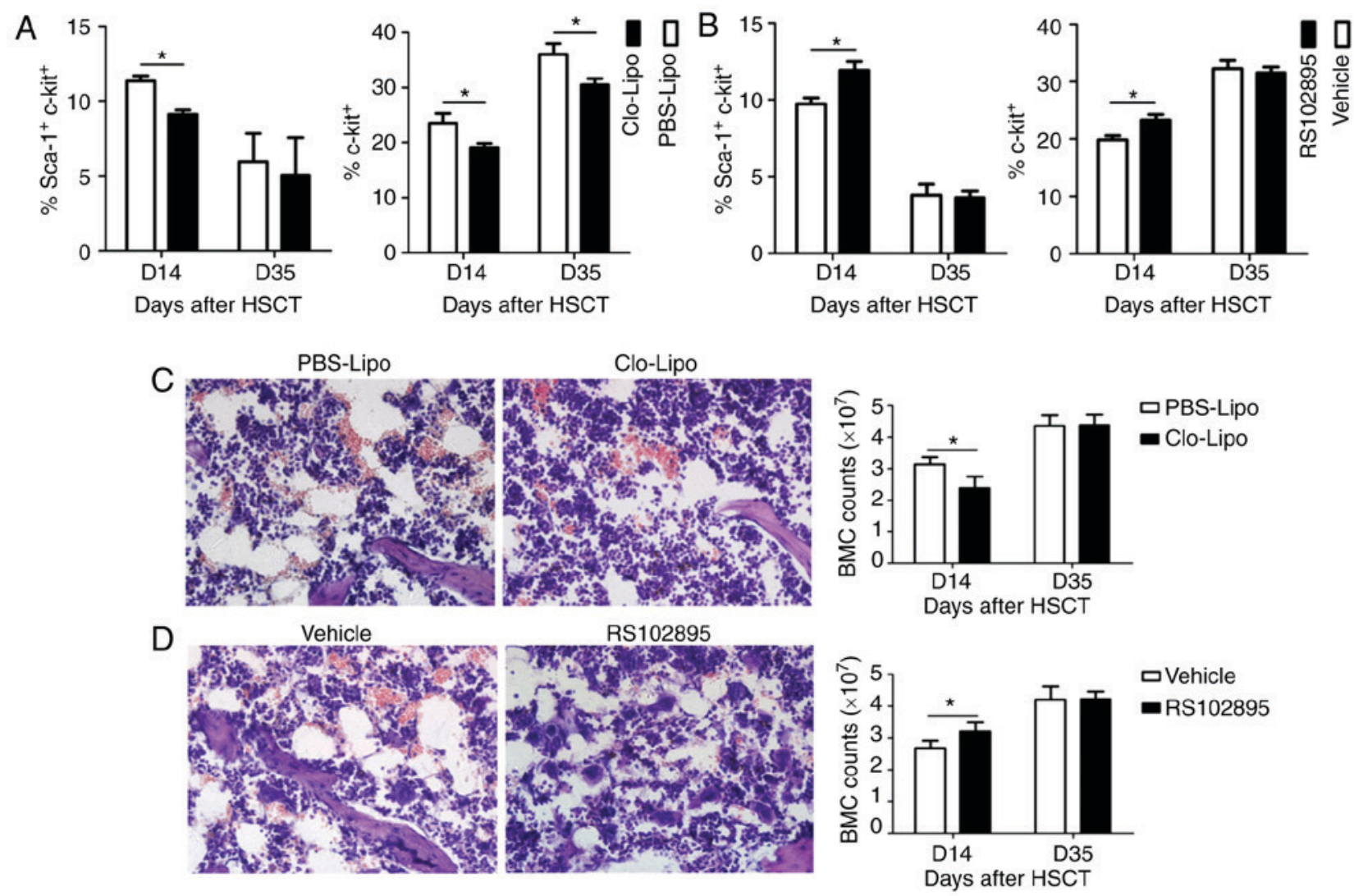

Figure 4. Hematopoietic stem/progenitor cells and bone marrow injury in mice after treatment with Clo-Lipo or RS102895 following HSCT. On D14 and D35 post transplantation, bone marrow cells were isolated from mice treated with Clo-Lipo (A) or RS102895 (B) for the measurement of the number of c-kit ${ }^{+}$sca ${ }^{+}$ stem cells and c-kit ${ }^{+}$progenitor cells by flow cytometry. In addition, on D14 and D35 post transplantation, bilateral femur and tibia were isolated from mice treated with Clo-Lipo (C) or RS102895 (D) for analysis of bone marrow pathology by H\&E staining and number of bone marrow cells (magnification, x400). ${ }^{*} \mathrm{P}<0.05$ ( $\left.\mathrm{n}=4\right)$. Clo-Lipo, Clodronate Liposomes; HSCT, hematopoietic stem cell transplantation; PBS-Lipo, PBS Liposomes.

by how macrophages promote peripheral hematopoiesis after transplantation remains unclear and requires further investigations.

Given altered hematopoietic reconstitution after manipulation of macrophages number, hematopoietic stem/progenitor cells were also measured. Consistent with slower hematopoietic recovery, numbers of hematopoietic stem and progenitor cells were significantly lower in Clo-Lipo-treated mice on D14 compared with PBS-Lipo (Fig. 4A). Even the number of hematopoietic progenitor cells was increased on D35, it was still lower in mice treated with Clo-Lipo than those treated with PBS-Lipo (Fig. 4A). In terms of RS102895-treated mice, numbers of hematopoietic stem/progenitor cells were significantly higher on D14 compared with Vehicle $(\mathrm{P}<0.05)$ (Fig. 4B). These data indicated that altered numbers of hematopoietic stem and progenitor cells might account for the different hematopoietic reconstitution after manipulation of macrophages.

In order to evaluate the pathology changes of bone marrow after manipulation of macrophages, $\mathrm{H} \& \mathrm{E}$ staining of bone marrow was performed on D14 post HSCT. As seen in Fig. 4C, hemorrhage and cavity were observed in Clo-Lipo and PBS-Lipo group with more severe in Clo-Lipo-treated mice. In addition, number of bone marrow cells appeared to be lower in mice treated with Clo-Lipo than PBS-Lipo. However, all the above pathologic damages of bone marrow were ameliorated in mice after RS102895 treatment (Fig. 4D) with increased number of bone marrow cells. These data indicated that macrophages might play a protective role during bone marrow injury after transplantation. However, the exact mechanism by how macrophages ameliorate bone marrow injury after HSCT remains unclear and awaits investigation in the future.

The potential limitation of our study was that the mechanism by which macrophages influence the hematopoietic cells was not investigated, which might be through affecting bone marrow microenvironment as demonstrated by ameliorated bone marrow injury and increased bone marrow cell number after increasing macrophages in bone marrow after HSCT (Fig. 4C and D). We plan to investigate it in the future.

In conclusion, out study demonstrated that bone marrow macrophages play a protective role in the inflammatory injury of bone marrow and accelerate hematopoietic reconstitution in mice after HSCT, suggesting manipulation of macrophages might be a novel approach for improving the efficacy of HSCT.

\section{Acknowledgements}

Not applicable.

\section{Funding}

The present study was supported by National Natural Science Foundation of China (grant nos. 81370602, 81400082 and 81570096), the Natural Science Foundation of Jiangsu 
Province (grant no. BK20140219) and the Jiangsu University Excellent Science and Technology Innovation Team.

\section{Availability of data and materials}

All data generated or analyzed during the present study are included in this published article.

\section{Authors' contributions}

JQ, LL, YX, WJ, PZ, YJ, ML, WL, LD, YW, KQ, and DL performed the experiments and analyzed the data. XZ analyzed the data. KX and LZ designed the study and co-wrote the manuscript.

\section{Ethics approval and consent to participate}

All experimental procedures involving animals were approved by the Ethics Committee of Xuzhou Medical University.

\section{Consent for publication}

Not applicable.

\section{Competing interests}

The authors declare that they have no competing interests.

\section{References}

1. Vyas P, Appelbaum FR and Craddock C: Allogeneic hematopoietic cell transplantation for acute myeloid leukemia. Biol Blood Marrow Transplant 21: 8-15, 2015.

2. Garcia IN: Role of hematopoietic stem cell transplantation in multiple myeloma. Clin Lymphoma Myeloma Leuk 15: 86-91, 2015.

3. Danby R and Rocha V: Improving engraftment and immune reconstitution in umbilical cord blood transplantation. Front Immunol 5: 68, 2014.

4. Charbord P: Hemopoietic stem cells: Analysis of some parameters critical for engraftment. Stem Cells 12: 545-562, 1994.

5. Chang L, Frame D, Braun T, Gatza E, Hanauer DA, Zhao S, Magenau JM, Schultz K, Tokala H, Ferrara JL, et al: Engraftment syndrome after allogeneic hematopoietic cell transplantation predicts poor outcomes. Biol Blood Marrow Transplant 20 $1407-1417,2014$

6. Morrison SJ and Scadden DT: The bone marrow niche for haematopoietic stem cells. Nature 505: 327-334, 2014
7. Yin $\mathrm{T}$ and Li L: The stem cell niches in bone. J Clin Invest 116 : 1195-1201, 2006.

8. Sison EA and Brown P: The bone marrow microenvironment and leukemia: Biology and therapeutic targeting. Expert Rev Hematol 4: 271-283, 2011.

9. Hume DA: The mononuclear phagocyte system. Curr Opin Immunol 18: 49-53, 2006.

10. Chow A, Lucas D, Hidalgo A, Méndez-Ferrer S, Hashimoto D, Scheiermann C, Battista M, Leboeuf M, Prophete C, van Rooijen $\mathrm{N}$, et al: Bone marrow CD169+ macrophages promote the retention of hematopoietic stem and progenitor cells in the mesenchymal stem cell niche. J Exp Med 208: 261-271, 2011.

11. Christopher MJ, Rao M, Liu F, Woloszynek JR and Link DC: Expression of the G-CSF receptor in monocytic cells is sufficient to mediate hematopoietic progenitor mobilization by G-CSF in mice. J Exp Med 208: 251-260, 2011.

12. Winkler IG, Sims NA, Pettit AR, Barbier V, Nowlan B, Helwani F, Poulton IJ, van Rooijen N, Alexander KA, Raggatt LJ and Lévesque JP: Bone marrow macrophages maintain hematopoietic stem cell (HSC) niches and their depletion mobilizes HSCs. Blood 116: 4815-4828, 2010.

13. Ludin A, Itkin T, Gur-Cohen S, Mildner A, Shezen E, Golan K, Kollet O, Kalinkovich A, Porat Z, D'Uva G, et al: Monocytes-macrophages that express $\alpha$-smooth muscle actin preserve primitive hematopoietic cells in the bone marrow. Nat Immunol 13: 1072-1082, 2012.

14. Qiao J, Wu J, Li Y, Xia Y, Chu P, Qi K, Yan Z, Yao H, Liu Y, $\mathrm{Xu} \mathrm{K}$ and Zeng L: Blockage of caspase-1 activation ameliorates bone marrow inflammation in mice after hematopoietic stem cell transplantation. Clin Immunol 162: 84-90, 2016.

15. Arango Duque G and Descoteaux A: Macrophage cytokines: Involvement in immunity and infectious diseases. Front Immunol 5: 491, 2014.

16. Poon IK, Lucas CD, Rossi AG and Ravichandran KS: Apoptotic cell clearance: Basic biology and therapeutic potential. Nat Rev Immunol 14: 166-180, 2014.

17. Zeng L, Ding S, Yan Z, Chen C, Sang W, Cao J, Cheng H and $\mathrm{Xu} \mathrm{K}$ : Irradiation induces homing of donor endothelial progenitor cells in allogeneic hematopoietic stem cell transplantation. Int J Hematol 95: 189-197, 2012.

18. Chen W, Li M, Su G, Zang Y, Yan Z, Cheng H, Pan B, Cao J, Wu Q, Zhao K, et al: Co-transplantation of hematopoietic stem cells and Cxcr4 Gene-transduced mesenchymal stem cells promotes hematopoiesis. Cell Biochem Biophys 71: 1579-1587, 2015.

19. van Rooijen $\mathrm{N}$ and Hendrikx E: Liposomes for specific depletion of macrophages from organs and tissues. Methods Mol Biol 605: 189-203, 2010

This work is licensed under a Creative Commons Attribution-NonCommercial-NoDerivatives 4.0 International (CC BY-NC-ND 4.0) License. 